M. EGUCHI AND Y. KIJIMA

KÖDAI MATH. SEM. REP.

26 (1975), 109-112

\title{
A NOTE ON SEMIGROUPS OF MARKOV OPERATORS ON $C(X)$
}

\author{
By Masayoshi Eguchi and Yōichi KiJima
}

\section{Introduction.}

Let $X$ be a compact Hausdorff space, and let $C(X)$ be the commutative $C^{*}$. algebra of all continuous complex functions on $X$. A bounded linear operator $T$ of $C(X)$ into itself is called a Markov operator it $T \geqq 0,\|T\|=1$, and $T 1=1$.

Let $\Sigma$ be a semigroup of Markov operators. For each $f \in C(X), \overline{c 0}\{T f: T \in \Sigma\}$ denotes the closed convex hull of $\{T f: T \in \Sigma\} . g \in C(X)$ is called a $\Sigma$-invariant function if $T g=g$ for all $T \in \Sigma$.

In ergodic theory the following conditions on $\Sigma$ are interesting: (I) Each $\overline{\mathrm{co}}\{T f: T \in \Sigma\}$ contains exactly one $\Sigma$-invariant function. (II) Each $\overline{\mathrm{co}}\{T f: T \in \Sigma\}$ contains at least one $\Sigma$-invariant function. In Theorem 1 , we shall give some necessary and sufficient conditions that (I) holds.

Let $C(X)^{*}$ be the dual Banach space of $C(X) . \mu \in C(X)^{*}$ is called a state if $\mu \geqq 0$ and $\|\mu\|=\mu(1)=1$. If $T$ is a Markov operator and if $\mu$ is a state, then $T^{*} \mu$ is also a state where $T^{*}$ denotes the adjoint operator of $T$. A state $\mu$ is called a $\Sigma$-invariant state if $T^{*} \mu=\mu$ for all $T \in \Sigma$.

Let $K_{\Sigma}$ be the set of all $\Sigma$-invariant states. Then $K_{\Sigma}$ is a weak*-compact convex subset of $C(X)^{*} . \mu \in K_{\Sigma}$ is called an extremal $\Sigma$-invariant state if $\mu$ is an extreme point of $K_{\Sigma}$.

A proper closed ideal $I$ of $C(X)$ is called a $\Sigma$-invariant ideal if $T(I) \subset I$ for all $T \in \Sigma$. There exists at least one maximal $\Sigma$-invariant ideal, and each $\Sigma$ invariant ideal is contained in some maximal $\Sigma$-invariant ideal. If $\mu$ is a $\Sigma$. invariant state, then $I_{\mu}=\{f \in C(X): \mu(|f|)=0\}$ is a $\sum$-invariant ideal.

In Theorem 2, we shall show that if (I) holds, then $\mu \rightarrow I_{\mu}$ is a bijection of the set of all extremal $\Sigma$-invariant states onto the family of all maximal $\Sigma$ invariant ideals.

Our discussion is much due to Deleeuw and Glicksberg [1], Schaefer [2], Sine [3], and Takahashi [4].

\section{Theorems.}

$\operatorname{co} \Sigma$ denotes the set of all finite convex linear combinations of operators in $\Sigma$. $\operatorname{co} \Sigma$ is also a semigroup of Markov operators. We note that $\overline{\mathrm{co}}\{T f$ :

Received May 17, 1973. 
$T \in \Sigma\}=\{\overline{A f: A \in \operatorname{co} \Sigma}\} . \quad \tilde{f}$ denotes the unique $\Sigma$-invariant function in $\overline{\mathrm{co}}\{T f$ : $T \in \Sigma\}$ whenever (I) holds.

LEMMA 1. If (I) holds, then for any $\varepsilon>0$ and $f_{i} \in C(X)(i=1,2, \cdots, n)$, there exists an $A \in \operatorname{co} \Sigma$ such that $\left\|\tilde{f}_{i}-A f_{i}\right\| \leqq \varepsilon(\imath=1,2, \cdots, n)$.

Proof. It is easy to see that $\widetilde{A f}=\tilde{f}$ for all $f \in C(X)$ and $A \in \operatorname{co} \Sigma$. First we choose an $A_{1} \in \operatorname{co} \Sigma$ such that $\left\|\tilde{f}_{1}-A_{1} f_{1}\right\| \leqq \varepsilon$. Next we choose an $A_{2} \in \operatorname{co} \Sigma$ such that $\left\|\widetilde{A_{1} f_{2}}-A_{2}\left(A_{1} f_{2}\right)\right\| \leqq \varepsilon$. Let $A=A_{2} A_{1}$. Then $A \in \operatorname{co} \Sigma$ and $\left\|\tilde{f}_{i}-A f_{i}\right\| \leqq \varepsilon(i=1,2)$. An induction argument completes the proof.

Let $B(\Sigma)$ be the commutative $C^{*}$-algebra of all bounded complex functions on $\Sigma$. For each $f \in C(X)$ and $\nu \in C(X)^{*}$, we define $f \otimes \nu \in B(\Sigma)$ by $(f \otimes \nu)(T)=$ $\nu(T f)$. Let $L(\Sigma)$ be the linear span of $\left\{f \otimes \nu: f \in C(X), \nu \in C(X)^{*}\right\}$ in $B(\Sigma)$. We note that $1 \in L(\Sigma)$ and $\varphi^{*} \in L(\Sigma)$ if $\varphi \in L(\Sigma)$ where $\varphi^{*}$ denotes the complex conjugate function of $\varphi$, and that $\varphi_{s}\left(\right.$ or $\left.{ }_{s} \varphi\right) \in L(\Sigma)$ if $S \in \Sigma$ and $\varphi \in L(\Sigma)$ where $\varphi_{s}$ (or ${ }_{s} \varphi$ ) denotes the right (or left) translation of $\varphi$ by $S$. $m \in L(\Sigma)^{*}$ is called a right (or left) invariant mean on $L(\Sigma)$ if $m(\varphi) \geqq 0$ whenever $\varphi \geqq 0,\|m\|=m(1)=1$, and $m\left(\varphi_{s}\right)$ (or $\left.m\left({ }_{s} \varphi\right)\right)=m(\varphi)$ for all $S \in \Sigma$ and $\varphi \in L(\Sigma)$. A right and left invariant mean $m$ on $L(\Sigma)$ is called a two-sided invariant mean on $L(\Sigma)$. If $m$ is a right invariant mean on $L(\Sigma)$, then for each state $\mu$ we can define $\tilde{\mu} \in K_{\Sigma}$ by $\tilde{\mu}(f)=$ $m(f \otimes \mu)$. In the following theorem, $M_{\Sigma}$ denotes the set of all $\Sigma$-invariant functions in $C(X)$.

THEOREM 1. The following conditions are equivalent.

(1) (I) holds.

(2) There exists a two-sided invariant mean on $L(\Sigma)$, and $M_{\Sigma}$ separates $K_{\Sigma}$.

(3) There exists a right invariant mean on $L(\Sigma)$, and $M_{\Sigma}$ separates $K_{\Sigma}$.

(4) There exists a right invariant mean on $L(\Sigma)$, and (II) holds.

Proof. (1) implies (2): If $\mu_{1}$ and $\mu_{2}$ are distinct $\Sigma$-invariant states, then $\mu_{1}(f) \neq \mu_{2}(f)$ for some $f \in C(X)$. This implies that $\mu_{1}(\tilde{f})=\mu_{1}(f) \neq \mu_{2}(f)=\mu_{2}(\tilde{f})$. Thus $M_{\Sigma}$ separates $K_{\Sigma}$. For each $\varphi=\sum_{i=1}^{n} f_{i} \otimes \nu_{i} \in L(\Sigma)$, we define $m(\varphi)=\sum_{i=1}^{n} \nu_{i}\left(\tilde{f}_{2}\right)$. We shall show that $m(\varphi)$ is independent of the particular representation of $\varphi$ and that $m$ is a two-sided invariant mean on $L(\Sigma)$. Suppose $\sum_{i=1}^{n} f_{i} \otimes \nu_{i}$ is identically zero. By Lemma 1 , for any $\varepsilon>0$ there exists an $A \in \operatorname{co} \Sigma$ such that $\left\|\tilde{f}_{i}-A f_{i}\right\| \leqq \varepsilon(i=1,2, \cdots, n)$. Then we have

$$
\left|\sum_{i=1}^{n} \nu_{i}\left(\tilde{f}_{\imath}\right)\right| \leqq\left|\sum_{i=1}^{n} \nu_{i}\left(\tilde{f}_{i}-A f_{\imath}\right)\right|+\left|\sum_{i=1}^{n} \nu_{i}\left(A f_{\imath}\right)\right| \leqq\left(\sum_{i=1}^{n}\left\|\nu_{i}\right\|\right) \varepsilon .
$$

Since $\varepsilon>0$ is arbitrary, $\sum_{i=1}^{n} \nu_{i}\left(\tilde{f}_{\imath}\right)=0$. Thus we may unambiguously define $m(\varphi)$. It is easy to see that $m$ is linear and $m(1)=1$. We shall show that $\|m\|=1$. Since $m(1)=1$, it suffices to show that $\|m\| \leqq 1$. Suppose $\varphi=\sum_{r=1}^{n} f_{i} \otimes \nu_{i}$. Again by Lemma 1 , for any $\varepsilon>0$ there exists an $A \in \operatorname{co} \Sigma$ such that $\left\|\tilde{f}_{i}-A f_{i}\right\| \leqq \varepsilon(i=$ $1,2, \cdots, n)$. Then we have 


$$
|m(\varphi)| \leqq\left|\sum_{\imath=1}^{n} \nu_{\imath}\left(\tilde{f}_{i}-A f_{\imath}\right)\right|+\left|\sum_{\imath=1}^{n} \nu_{i}\left(A f_{\imath}\right)\right| \leqq\left(\sum_{i=1}^{n}\left\|\nu_{i}\right\|\right) \varepsilon+\|\varphi\| \text {. }
$$

Since $\varepsilon>0$ is arbitrary, $|m(\varphi)| \leqq\|\varphi\|$. It is easy to see that $m\left(\varphi^{*}\right)=\overline{m(\varphi)}$. Suppose $\varphi=\sum_{\imath=1}^{n} f_{i} \otimes \nu_{\imath} \geqq 0$. Then $m(\varphi)$ is real. We assume that $\alpha=m(\varphi)<0$. Let $\varepsilon$ be a number such that $0<\varepsilon<-\alpha$. By Lemma 1, we can choose an $A \in \operatorname{co} \Sigma$ such that $\left\|\tilde{f}_{i}-A f_{i}\right\| \leqq \varepsilon / \sum_{\imath=1}^{n}\left\|\nu_{\imath}\right\|(\imath=1,2, \cdots, n)$. Let $\beta=\sum_{i=1}^{n} \nu_{i}\left(A f_{\imath}\right)$. Then $\beta \geqq 0$ and $|\alpha-\beta|<\varepsilon$, so we have $0 \leqq \beta=|\alpha-\beta|+\alpha \leqq \varepsilon+\alpha<0$. This is a contradiction. Thus $m(\varphi)>0$. If $\varphi=\sum_{\imath=1}^{n} f_{\imath} \otimes \nu_{i}$, then $\varphi_{s}=\sum_{\imath=1}^{n} S f_{i} \otimes \nu_{\imath}$ and ${ }_{s} \varphi=\sum_{\imath=1}^{n} f_{i} \otimes S *_{\nu_{i}}$. It is easy to see that $m\left(\varphi_{s}\right)=m\left({ }_{s} \varphi\right)=m(\varphi)$.

(2) implies (3): Evident.

(3) implies (4): Let $m$ be a right invariant mean on $L(\Sigma)$, and let $\delta_{x}$ be the point measure at $x \in X$. For each $f \in C(X)$, we can define $P f \in C(X)$ by $(P f)(x)=m\left(f \otimes \delta_{x}\right)$ (see [3] and [4]). Then $P$ is a Markov operator such that $P T=P$ for all $T \in \Sigma$ and $P g=g$ for all $g \in M_{\Sigma}$. We shall show that $P f$ is a $\Sigma$ invariant function in $\overline{c o}\{T f: T \in \Sigma\}$. Let $\mu$ be a state. Then $P^{*} T^{*} \mu, P^{*} \mu$ and $\tilde{\mu}$ are $\Sigma$-invariant states. If $g$ is a $\Sigma$-invariant function, then $\left(P^{*} T^{*} \mu\right)(g)=$ $\mu(T P g)=\mu(T g)=\mu(g),\left(P^{*} \mu\right)(g)=\mu(P g)=\mu(g)$, and $\tilde{\mu}(g)=m(g \otimes \mu)=\mu(g)$. Since $M_{\Sigma}$ separates $K_{\Sigma}$, we have $P^{*} T^{*} \mu=P^{*} \mu=\tilde{\mu}$, which implies that $T P=P$ for all $T \in \Sigma$ and $\nu(P f)=m(f \otimes \nu)$ for all $f \in C(X)$ and $\nu \in C(X)^{*}$. Thus $P f$ is a $\Sigma$ invariant function. If $P f$ is not contained in $\overline{c o}\{T f: T \in \Sigma\}$, there exists a $\nu \in C(X)^{*}$ such that $\sup \{\Re \geq \nu(T f): T \in \Sigma\}<\Re e \nu(P f)$, but Re $\nu(P f)=\Re e(f \otimes \nu)=$ $m(\Re e(f \otimes \nu)) \leqq \sup \{\Re e \nu(T f): T \in \Sigma\}$. This is a contradiction.

(4) implies (1): The proof is similar to [4].

THEOREM 2. If (I) holds, then $\mu \rightarrow I_{\mu}$ is a bijection of the set of all extremal

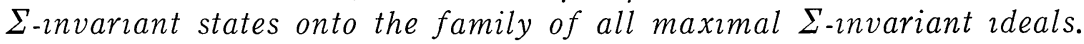

Proof. Let $I$ be a maximal $\Sigma$-invariant ideal. As well known, there exists an $x_{0} \in X$ such that any function in $I$ vanishes at $x_{0}$. For each $f \in C(X)$ we define $\mu(f)=\tilde{f}\left(x_{0}\right)$, then $\mu$ is a $\sum$-invariant state which vanishes on $I$. The Schwarz inequality $\mu(|f|) \leqq \sqrt{\mu\left(|f|^{2}\right)}$ implies that $I \subset I_{\mu}$ and therefore $I=I_{\mu}$. Let $K_{\Sigma, I}=\left\{\mu \in K_{\Sigma}: I=I_{\mu}\right\}$, then $K_{\Sigma, I}$ is a nonempty weak*-compact convex subset of $C(X)^{*}$. By the Krein-Milman theorem there exists an extreme point $\mu_{0}$ of $K_{\Sigma, I}$. It is easy to see that $\mu_{0}$ is also an extreme point of $K_{\Sigma}$.

Let $\mu$ be an extremal $\Sigma$-invariant state. If $I_{\mu}$ is not maximal, then there exists a maximal $\Sigma$-invariant ideal $I$ containing $I_{\mu}$. We can choose a $\Sigma$-invariant function $g$ from $I-I_{\mu}$ such that $0 \leqq g \leqq 1$ and $0<\mu(g)<1$. Let $\mu_{1}(f)=\mu(\tilde{f} g) / \mu(g)$ and $\mu_{2}(f)=\mu(\tilde{f}(1-g)) / \mu(1-g)$. Then $\mu_{1}$ and $\mu_{2}$ are $\Sigma$-invariant states, and $\mu=$ $\alpha \mu_{1}+(1-\alpha) \mu_{2}$ where $\alpha=\mu(g)$. Since $\mu$ is extremal, $\mu_{1}=\mu_{2}$ and therefore $\mu_{1}(g)$ $=\mu_{2}(g)$, which implies $\mu\left(g^{2}\right)=(\mu(g))^{2}$. It follows easily from the Schwarz inequality that $\mu(|g-\mu(g) 1|)=0$. This shows that $g-\mu(g) 1 \in I$ and therefore $1 \in I$. This is a contradiction.

Let $\mu_{1}$ and $\mu_{2}$ be distinct extremal $\Sigma$-invariant states. Then there exists a $\Sigma$-invariant function $g$ such that $0 \leqq g \leqq 1$ and $\mu_{1}(g) \neq \mu_{2}(g)$. If $I_{\mu_{1}}=I_{\mu_{2}}$, then 
$0<\mu_{1}(g)<1$. Let $\mu_{3}(f)=\mu_{1}(\tilde{f} g) / \mu_{1}(g)$ and $\mu_{4}(f)=\mu_{1}(\tilde{f}(1-g)) / \mu_{1}(1-g)$. Then $\mu_{3}$ and $\mu_{4}$ are $\Sigma$-invariant states, and $\mu_{1}=\alpha \mu_{3}+(1-\alpha) \mu_{4}$ where $\alpha=\mu_{1}(g)$. Since $\mu_{1}$ is extremal, $\mu_{3}=\mu_{4}$. As in the above paragraph, it follows that $g-\mu_{1}(g) 1 \in I_{\mu_{1}}$ and therefore $g-\mu_{1}(g) 1 \in I_{\mu_{2}}$, which implies that $\mu_{1}(g)=\mu_{2}(g)$. This is a contradiction. Thus we conclude that $I_{\mu_{1}}$ and $I_{\mu_{2}}$ are distinct.

\section{REFERENCES}

[1] Deleeuw, K., And I. Glicksberg, Applications of almost periodic compact1fications. Acta Math. 105 (1961), 65-97.

[2] Schaefer, H.H., Invariant ideals of positive operators in $C(X)$. Illinois J. Math. 12 (1968), 525-538.

[3] Sine, R., Geometric theory of a single Markov operator. Pacific J. Math. 27 (1968), 155-166.

[4] TAKAHASHI, W., Invariant ideals for amenable semigroups of Markov operators. Kōdai Math. Sem. Rep. 23 (1971), 121-126.

Central Research Institute of Electric Power Industry, and Tokyo Institute of Technology. 